\title{
Understanding Topological and Spatial Attributes of Bus Transportation Networks in Cities of Chongqing and Chengdu
}

\author{
Dan Wan $\mathbb{D}^{1},{ }^{1}$ Yong Huang $\mathbb{D}^{2,3}$ Jie Feng $\mathbb{D}^{4},{ }^{4}$ Yaling Shi, ${ }^{2}$ Kairui Guo $\mathbb{D}^{1},{ }^{1}$ and Ran Zhang $\mathbb{D}^{2}$ \\ ${ }^{1}$ Chongqing University Planning \& Design Institute Co. Ltd, Chongqing University, Chongqing 400030, China \\ ${ }^{2}$ Faculty of Architecture and Urban Planning, Chongqing University, Chongqing 400030, China \\ ${ }^{3}$ Key Laboratory of New Technology for Construction of Cities in Mountain Areas, Chongqing University, Chongqing 400030, China \\ ${ }^{4}$ Beijing Tsinghua Tongheng Urban Planning \& Design Institute Co. Ltd, Tsinghua University, Beijing 10085, China
}

Correspondence should be addressed to Yong Huang; hyong@cqu.edu.cn

Received 27 April 2018; Revised 12 August 2018; Accepted 30 September 2018; Published 17 October 2018

Academic Editor: Emilio Insfran Pelozo

Copyright (c) 2018 Dan Wan et al. This is an open access article distributed under the Creative Commons Attribution License, which permits unrestricted use, distribution, and reproduction in any medium, provided the original work is properly cited.

\begin{abstract}
It is critical to understand the characteristics of public transportation networks (PTNs). Existing studies have mainly focused on the topological structure of PTNs and have revealed the commonalities of the topological structures of PTNs. However, few studies have examined the differences regarding topological structure characteristics between the PTNs of different cities. In addition, the nature and extent of the influence of specific urban geographic conditions and morphology on PTNs are unclear. This paper focuses on the influence of urban spatial and geographic environments on bus transportation networks (BTNs) by comparatively studying the topological and spatial attributes of two typical BTNs, respectively, from a mountainous city and a plain city in China, from the perspectives of basic statistical properties, types, connection properties, and spatial attributes, by using the complex networks theory and spatial analysis method. The results reveal that the two BTNs have similar statistical properties and they both have scalefree features as well as small-world features. However, these two BTNs are significantly different in the connection properties and spatial attributes. The difference is found closely related to the city's geographic conditions and spatial morphology. The implications of this study regarding urban traffic planning and land planning are discussed.
\end{abstract}

\section{Introduction}

An in-depth understanding of the structure and topological properties of public transportation networks (PTNs) is important for urban planning and management, policymaking, and disaster-prevention and disaster-mitigation [16]. Complex network analysis (CNA), as a formalized methodology, is a mathematical form of graph theory used to characterize network structure and topological properties [713]. In recent years, CNA has been instrumental in studying PTNs, and considerable research has been conducted in fields such as bus transportation networks (BTNs) [1, 1417], aviation networks [2,18-20], metro networks [10, 21], railway networks [22], road networks [5], and maritime transportation networks [23]. The main research topics are as follows:

Continuous attention has been paid to the topological structure of PTNs, including the community properties [24] and the $k$-core layer [19], as well as the small-world and the scale-free features of networks [1, 22]. New indicators have been proposed, and new models have been established, or the existing models have been optimized. For instance, a dynamically weighted BTN model based on the average travel time [14] was developed, while a spatial route model of BTN based on competition and cooperation [25] was improved. The study of the robustness of the PTNs $[2,3,18$, 20 ] was conducted with discussing the dynamics of complex networks proposed by Albert [26]. Further studies regarding the relevant social dynamics of PTNs continued. For example, dissemination of diseases on BTNs [4] was analyzed. Analysis of the temporal variation of the PTNs has also been conducted. The factors influencing temporal variations in the aviation network over the period of a decade [2] were elucidated. Complex systems are often organized under the form of networks where nodes and edges are embedded in 
space. Many kinds of networks, such as the transportation networks, are all examples where space is relevant and where topology alone contains incomplete information [27]. Researchers also have studied the spatial attributes of PTNs in some aspects, such as fostering a new spatial representation model [16]. The results show (a) the connections between the geographic features of BTNs and the economic development conditions of certain areas [24], (b) the distribution rules of the key routes and nodes of BTNs [14], (c) the hierarchical geographic distribution rules of aviation networks [19], and (d) the relation between network structure of road and city form [5].

The above numerous studies have presented the commonality feature of the PTNs of various cities regarding their topological structures. It should be noted that PTNs are always manipulated by humans in specific geographic conditions, requiring that tasks such as transportation system management and urban planning should be implemented according to certain geographic environments. However, the effects on the statistical mechanics of PTNs caused by the urban geographic conditions and spatial morphology have not been elucidated.

Many researchers have demonstrated the scale-free feature of PTNs, such as Greek maritime network [23] and BTN in the city of Qingdao, China [14], indicating that scalefree feature serves as essential characteristics of many real networks [28]. Meanwhile, other studies have shown that the node degree distributions (NDD) of some PTNs comply with the exponential law, as exemplified by eight Polish cities including GOP [17] and the city of Harbin, China [15]. According to previous studies [29], if the NDD of a network complies with a power-law, i.e., the network has scale-free feature, then the new node is connected with the original network in a preferential arrangement. While if the NDD of a network complies with the rules of an exponential law, then the new node is connected to the original network at random. Therefore, it is necessary to carry out more studies to analyze the characteristics of NDD of urban PTNs and fully understand the statistical mechanics of how urban PTNs develop.

In this paper, data from a typical mountainous city, Chongqing, and a plain city, Chengdu (both in China), are collected to conduct a comparative analysis to characterize the topological structures and spatial attributes of BTNs to determine the effects on the statistical mechanics of development process of BTNs caused by the urban geographic conditions and spatial morphology. The mechanisms underlying the development and variation of the networks among different cities are also analyzed. Complex network theory and spatial analysis method, both already widely used to tackle the topology problems of real-life networks and examine the spatial structures of real-life geographic systems, respectively, are applied in this work.

The remainder of this paper is organized as follows. Section 2 describes the key methods used for data acquisition and research. Section 3 presents the results, and Section 4 presents a further discussion of the results. The last section provides a summary of suggestions for future research.

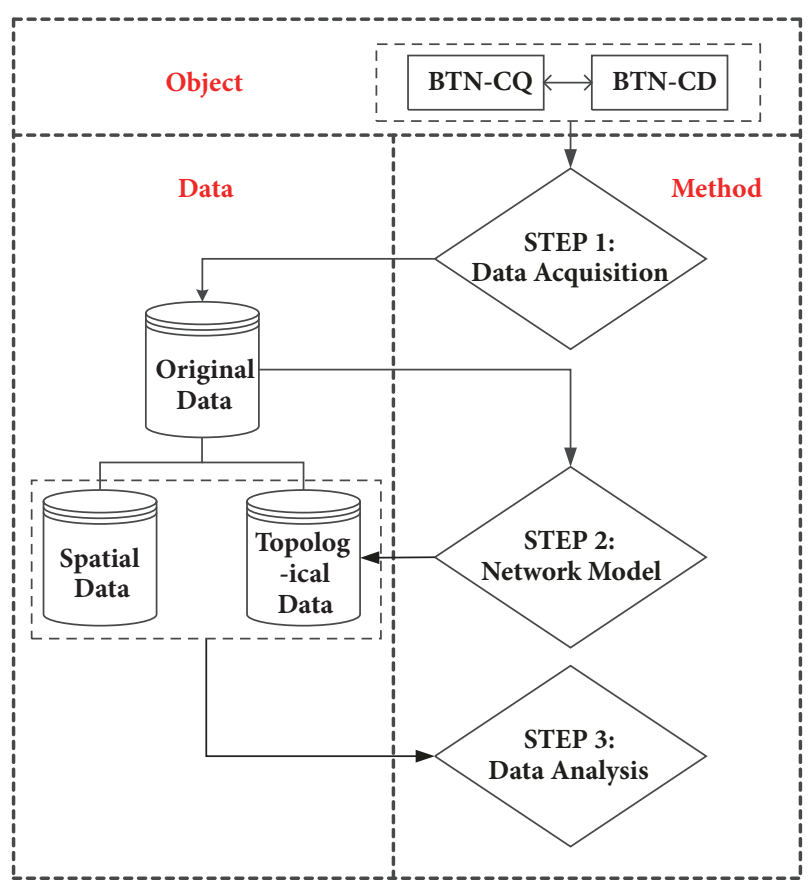

FIGURE 1: Framework of analysis.

\section{Data and Methods}

2.1. Overall Planning. A framework, as shown in Figure 1, is proposed to represent the analysis procedure for the efficient analysis of topological structure and spatial properties of the BTNs studied. The whole analysis procedure consists of the following three steps. Step 1, data acquisition: the original data of the bus routes (including stops contained in each route) with the information of geographic coordinate of each stop and geographic conditions of cities are obtained; Step 2, network models: the network models of BTNs to obtain the topology information are established; Step 3, data analysis: the data analysis is implemented. The details of these steps are presented in Sections 2.2-2.4.

2.2. Data Acquisition. The data of BTNs is obtained in Chongqing and Chengdu, as shown in Figure 2. They are two key cities where the bus transportation system serves as the main channel for intercity commuters in Southwest China.

In Chongqing, the major urban zones are within the range of the city expressway, which is also the case for Chengdu. There are considerable differences between the two cities in terms of geographic conditions and spatial morphology, as shown in Figure 3. ASTER GDEM (Advanced Spaceborne Thermal Emission and Reflection Radiometer Global Digital Elevation Model) was adopted in this paper. With its flat terrain, Chengdu is a plain city without wide rivers (Figure 3(a2)). In contrast, the main urban area of mountainous Chongqing has two large mountains and two major rivers, as shown in Figure 3(a1). Affected by the geographic condition, the spatial morphology of Chengdu presents a single-center circular structure; i.e., there is only one main center around which the surrounding areas develop 
TABLE 1: Bus transportation networks in Chongqing and Chengdu.

\begin{tabular}{|c|c|c|c|c|c|c|c|}
\hline study area & $\begin{array}{l}\text { terrain } \\
\text { feature }\end{array}$ & $\begin{array}{l}\text { urban spatial } \\
\text { morphology }\end{array}$ & $\begin{array}{l}\text { area } \\
\left(\mathrm{km}^{2}\right)\end{array}$ & $\begin{array}{c}\text { number of } \\
\text { routes }\end{array}$ & $\begin{array}{l}\text { number of } \\
\text { routes } / \mathrm{km}^{2}\end{array}$ & $\begin{array}{c}\text { number of } \\
\text { stops }\end{array}$ & $\begin{array}{l}\text { number of } \\
\text { stops } / \mathrm{km}^{2}\end{array}$ \\
\hline Chongqing & $\begin{array}{c}\text { Mountainous } \\
\text { region }\end{array}$ & $\begin{array}{c}\text { multi-center } \\
\text { spatial } \\
\text { structure }\end{array}$ & 2253 & 324 & 0.14 & 2539 & 1.13 \\
\hline Cheng-du & Plain region & $\begin{array}{l}\text { single-center } \\
\text { circular } \\
\text { structure }\end{array}$ & 597 & 515 & 0.86 & 2766 & 4.63 \\
\hline
\end{tabular}

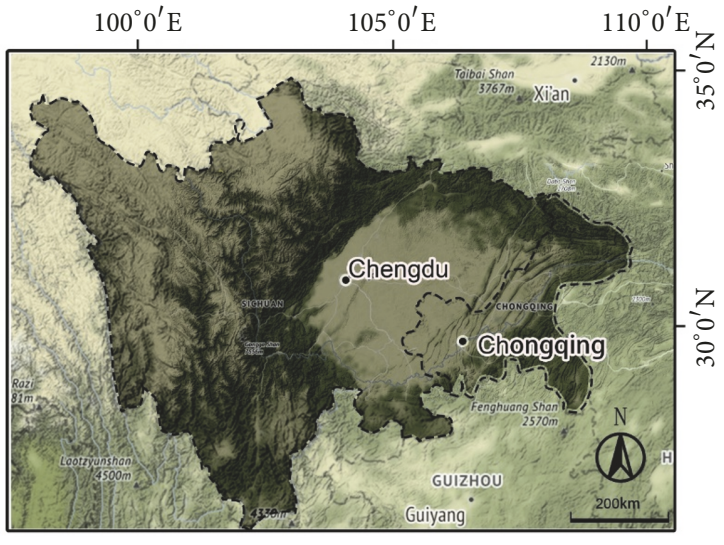

FIGURE 2: Map of locations of Chongqing and Chengdu in Southwest China.

in a ring-level (Figure 3(b2)). In contrast, in Chongqing, the urban spatial morphology exhibits a multicenter spatial structure with several centers and multiple relatively independent development groups, as shown in Figure 3(b1). The experimental dataset is offered by the 8684-bus website (http://www.8684.cn), and the locations of bus stations are shown in Figures 3(c1) and 3(c2). The observed period for which the BTNs were considered-routes and stops-starts on the 1st of October 2016 and ends on the 5th of October 2016. Although Chongqing covers a larger area than Chengdu, the two cities have similar numbers of bus stops (Table 1).

2.3. Network Models. Overall, there are three main network representations of BTNs: L-space, P-space, and C-space, illustrated in Figure 4. The details of network representations can be found in [37]. In this paper, the P-space representation is adopted because it effectively describes the transfer situations of bus transport system; a rational transfer organization of bus system is also indispensable to ensure the system work soundly and efficiently. According to the P-space representation, a station in the system is a node in the network model, and all stations in the same route are directly connected with one another to account for the presence of edges [22]. When the uplink route differs from the downlink route, the uplink route is adopted.

The network models are established with NetworkX (Version: 1.11) [38], a network analysis integrated package of
Python (Version: 3.5.2) (Figure 5). There are 2539 nodes and 80,301 edges in the bus transportation network of Chongqing (BTN-CQ), while there are 2,766 nodes and 92,641 edges in the bus transportation network of Chengdu (BTN-CD).

2.4. Data Analysis. In this section, a BTN analysis procedure is established to examine the BTNs in terms of four aspects, including basic statistical properties (i.e., density, average degree, average shortest path length, and clustering coefficient), types (i.e., scale-free feature and small-world feature), connection properties (i.e., distance distribution), and spatial attributes (i.e., degree distribution and Core layer distribution), as shown in Figure 6. The details of these aspects are presented in Sections 2.4.1-2.4.4.

\subsubsection{Basic Statistical Properties of Networks}

(1) Density. For a graph with $n$ nodes, the density $\rho$ shows the overall connectivity of the network nodes. The density $\rho$ is determined as follows [39]:

$$
\rho=\frac{2 m}{n(n-1)}
$$

where $m$ is the number of edges in a network.

(2) Average Degree. For a graph with $n$ nodes, the average degree $\langle k\rangle$ measures the number of average connections of a node and can be written as follows [40]:

$$
<k>\frac{1}{n} \sum_{i=1}^{n} k_{i}
$$

where $k_{i}$ is the degree of node $i$, i.e., the number of edges connected to node $i$.

(3) Average Shortest Path Length. For a graph with $n$ nodes, the average shortest path length $\langle l\rangle$ is the average shortest distance between two nodes. It can be written as [41]

$$
<l>=\frac{2}{n(n-1)} \sum_{i \leq j \leq n} d_{i j}
$$

where the shortest path length-distance $d_{i j}$ is the number of links in the shortest path between nodes $i$ and $j$.

(4) Clustering Coefficient. For a graph with $n$ nodes, the clustering coefficient was first introduced by Watts, D. J., and 


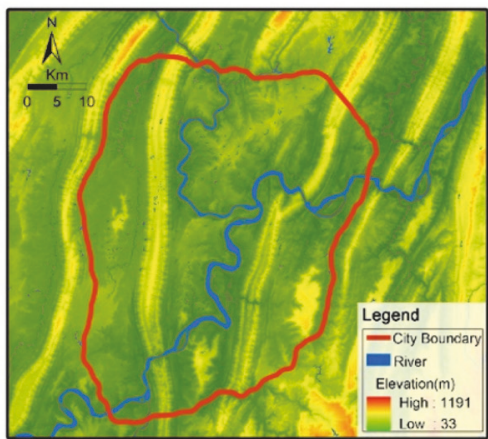

(a1)

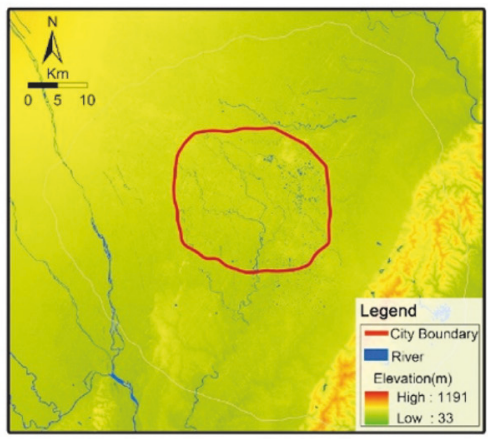

(a2)

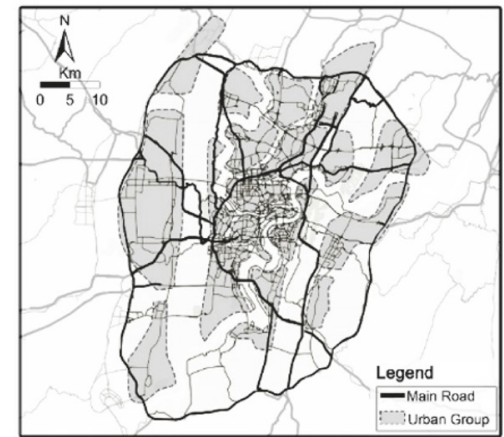

(b1)

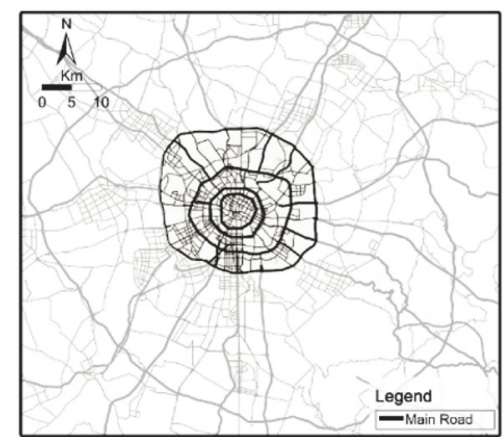

(b2)

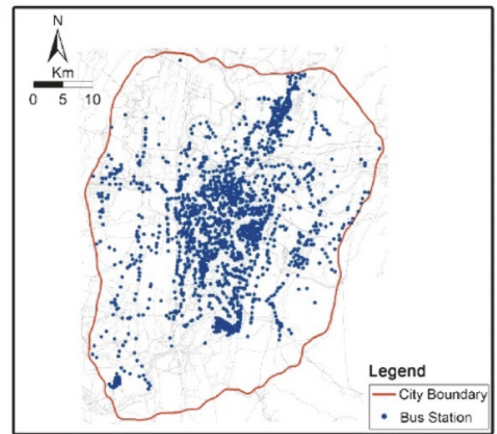

(c1)

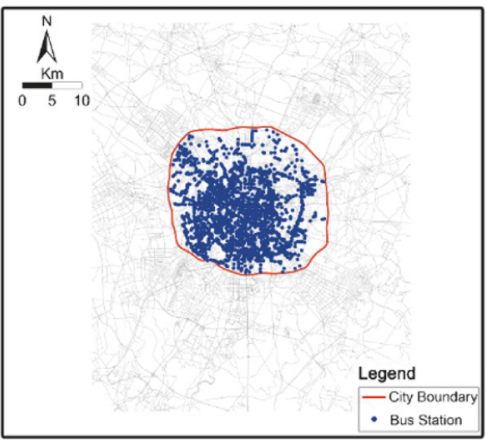

(c2)

FIgURE 3: Map of geographic condition, urban spatial morphology, and locations of bus stations. (al) geographic condition in Chongqing; (a2) geographic condition in Chengdu; (b1) urban spatial morphology in Chongqing; (b2) urban spatial morphology in Chengdu; (c1) locations of bus stations in Chongqing; (c2) locations of bus stations in Chengdu (employed in ArcGIS 10.2; Basemap: OpenStreetMap http://www.openstreetmap.org/).

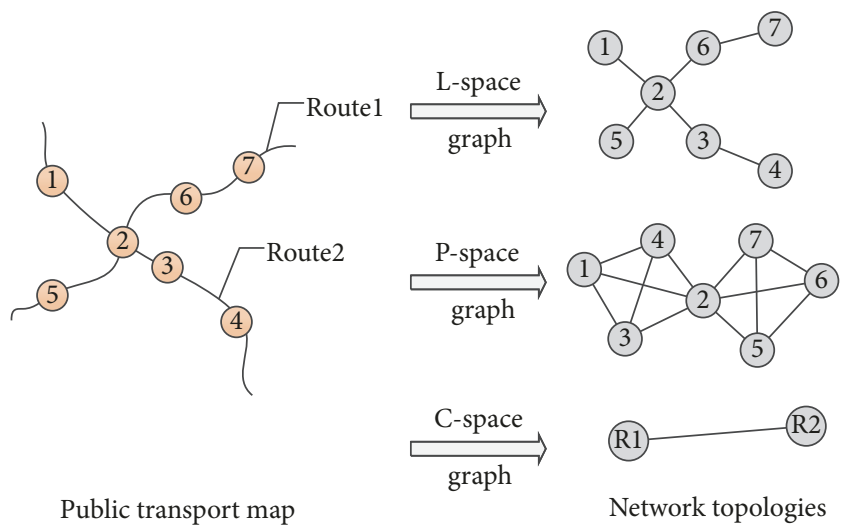

FIGURE 4: Public transport map and three typical network representations.

Strogatz, S. H. [41]. The clustering coefficient of node $i C_{i}$ is used to assess how close the neighbors of node $i$ are connected to each other.

$$
C_{i}=\frac{2 e_{i}}{k_{i}\left(k_{i}-1\right)}
$$

where $e_{i}$ is the number of the links between the neighbors of node $i$ and $k_{i}\left(k_{i}-1\right) / 2$ is the normalization factor equal to the maximum number of possible edges among the neighbors. In this work, the clustering coefficient $C C$, defined as the average clustering coefficient of all individual nodes, is applied.

$$
C C=\frac{1}{n} \sum_{i=1}^{n} C_{i}
$$

\subsubsection{Types of Networks}

(1) Scale-Free Feature. The node degree distribution $p(k)$, in which $p$ is the probability of $k$ edges for a randomly selected node, is adopted to measure the scale-free feature of a network [28, 39]. A scale-free feature exists in the network examined if $p(k)$ complies with the power-law distribution, meeting the linear relationship in the doublelogarithm coordinate system at the same time [39].

$$
\begin{aligned}
p(k) & =\mathrm{A} k^{-\gamma} \\
\ln p(k) & =-\gamma \ln k+\mathrm{c}
\end{aligned}
$$

where $\mathrm{A}, \gamma$, and $\mathrm{c}$ are constants.

The method of original node degree distribution (ONDD) was applied in the data-fitting process in many studies, and a number of networks with power-law degree distributions have been found [14, 23]. Meanwhile, it is valuable to compare the value of the correlation coefficient $\mathrm{R}^{2}$ calculated from the power-law fitting process and the value calculated from the exponential fitting process. 


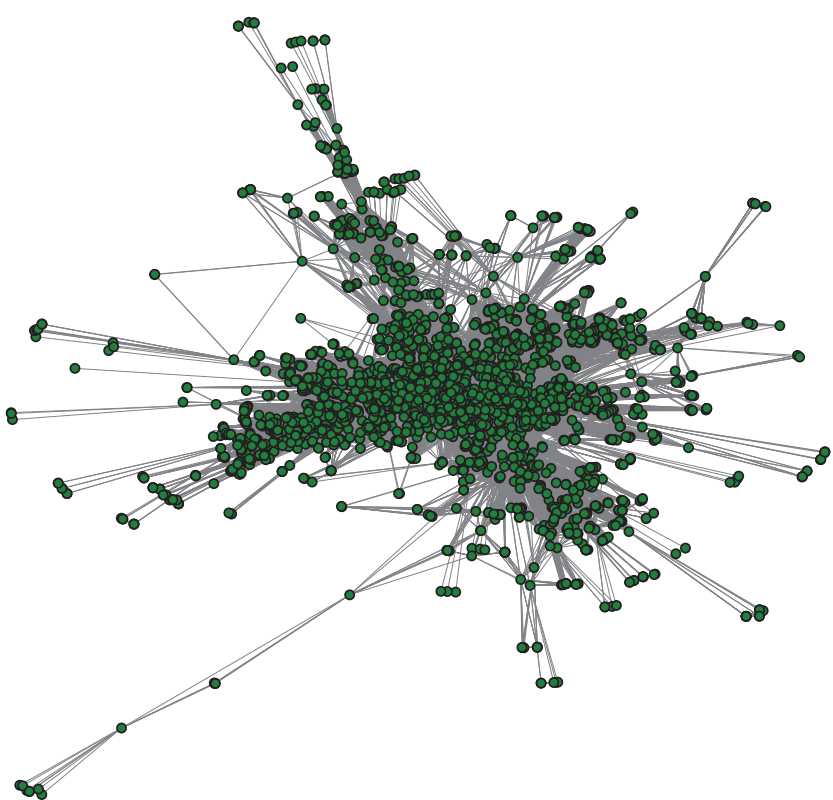

(a)

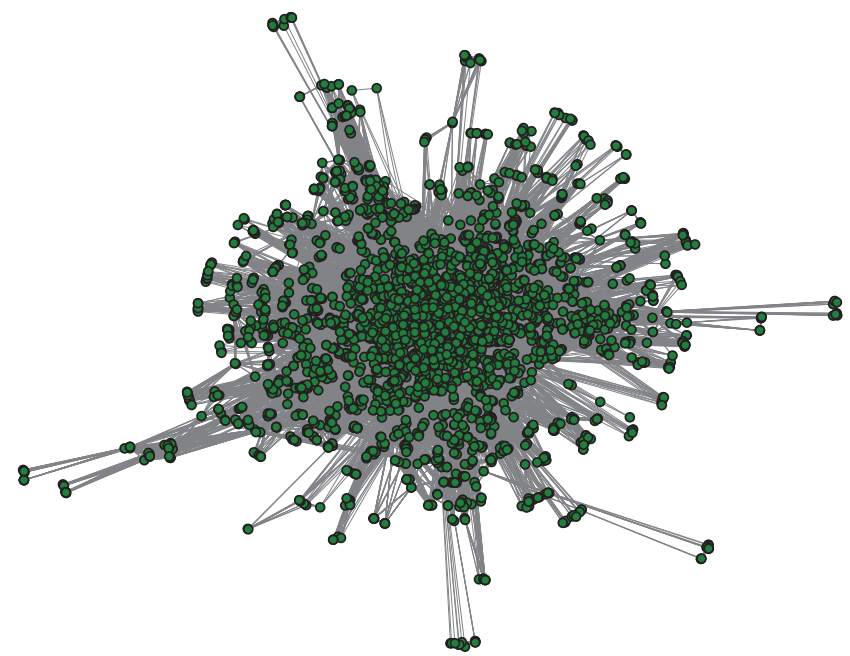

(b)

FIGURE 5: BTN's P-space networks as represented with NetworkX of (a) BTN-CQ and (b) BTN-CD.

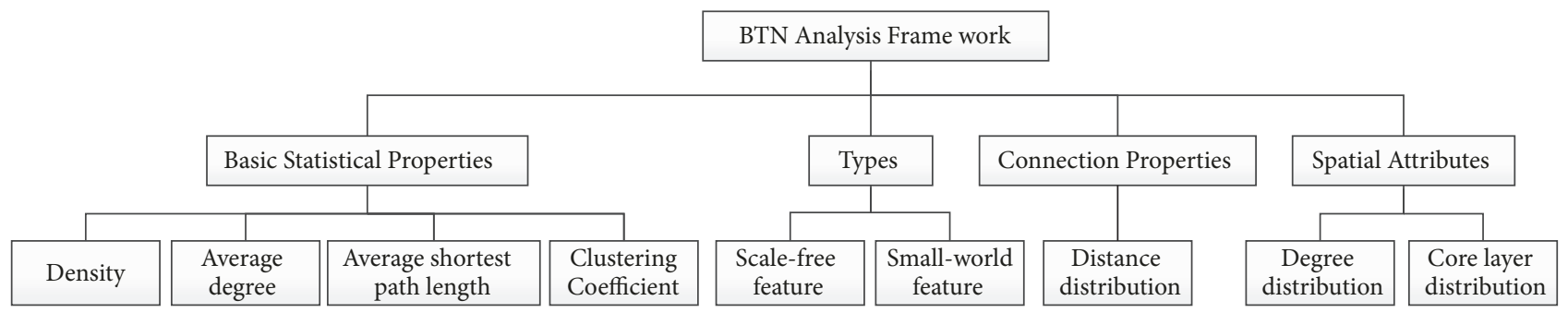

FIGURE 6: Analysis framework for research on BTNs.

Moreover, we compared the values of correlation coefficient $\mathrm{R}^{2}$ from the two BTNs observed and the randomly generated BA (Barabási-Albert) model, which is confirmed to have scale-free feature [29].

The BA model for comparison was generated by NetworkX, sharing a similar size to that of BTN-CQ and BTNCD (2,700 nodes and 85,376 edges for the BA model). The results of regression analysis on this model reveal that the ONDD indeed matches the power-law distribution and the correlation coefficient $\mathrm{R}^{2}$ of power-law is much higher than that of the exponential distribution shown in Figure 7 and Table 2 (0.8065 for power-law function and 0.5398 for exponential function).

(2) Small-World Feature. The small-world network was introduced by Watts and Strogatz [41]. Regular networks exhibit a relatively high clustering coefficient $C C$ and a relatively long average shortest path length $<l>$. By contrast, random networks exhibit a relatively low $C C$ and a relatively small $<l>$. The small-world network can be highly clustered (high clustering coefficient CC), like regular networks, yet have

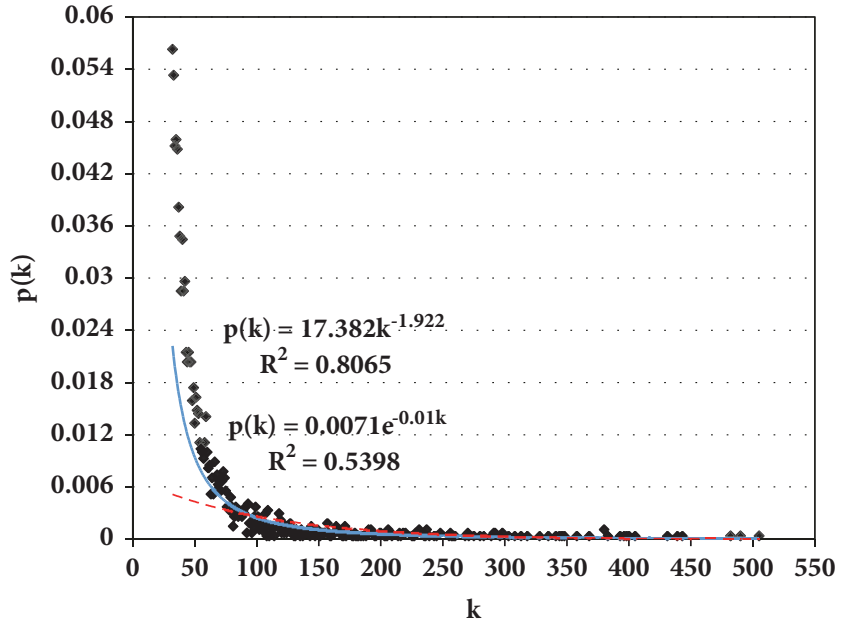

Figure 7: Curve fittings of the BA model by ONDD.

small characteristic path lengths (small average shortest path length $<l>$ ), like random networks [41]. 
TABLE 2: Curve fitting results of the BA model in the applications of ONDD.

\begin{tabular}{lccccrr}
\hline Network & Function & Type & Method & A & $\gamma$ & $\boldsymbol{\alpha}$ \\
\hline \multirow{2}{*}{ BA model } & $\mathrm{P}(k)=\mathrm{A} k^{-\gamma}$ & Power-law & \multirow{2}{*}{ ONDD } & 17.382 & 1.922 & 0.8065 \\
& $\mathrm{P}(k)=\mathrm{A} e^{-\alpha k}$ & Exponential & & 0.0071 & 0.5398 \\
\hline
\end{tabular}

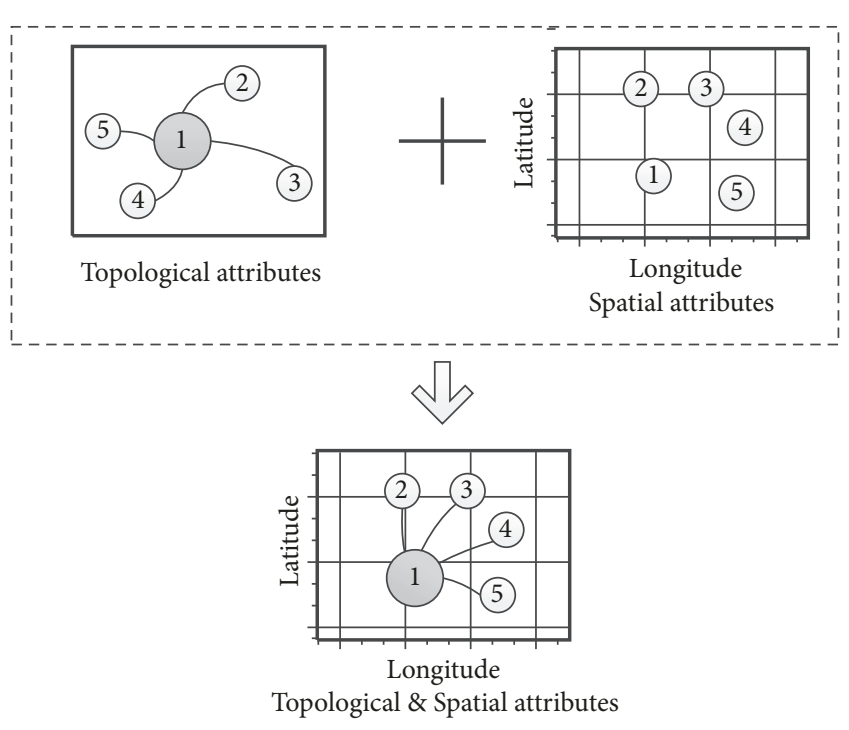

FIgURE 8: Illustration of combination of topological attributes and spatial attributes of networks.

2.4.3. Connection Properties of Networks. In the space-P model of BTNs, the shortest path length-distance $\left(d_{i j}\right)$ between nodes $i$ and $j$ can reflect the transfer times $\left(d_{i j^{-}}\right.$ 1) of BTNs for commuters shuttling between station $i$ and station $j$. To provide a better understanding of the connection properties of BTNs, it is valuable to get the distance distribution function, which reflects the probabilities of different distances between pairs of nodes chosen randomly.

2.4.4. Spatial Attributes of Networks. The special attributes of BTNs can be analyzed with the combination of the topological information, via degree and Core layer characteristics, and spatial coordinate information of bus stations (Figure 8). Degree $k_{i}$ represents the number of edges connected to node $i$. The $k$-core decomposition algorithm can be used to extract the Core layer (the most densely connected core of a network) $[19,42,43]$. The steps of the $k$-core algorithm are as follows: first, all nodes with degree $k_{i}$ equal to 1 are removed, and then, iteratively, the nodes with degrees of 2,3 , and so on $\left(k_{i}^{\prime}=t\right.$; note that $t$ increased by one every step from $k_{i}^{\prime}=1$ ) are also removed. Meanwhile, some of the new nodes with a lower degree than $t$ will also be removed in the course of an iteration ( $k_{i}^{\prime}$ is the degree of node $i$ after removing nodes in previous iterations). The algorithm stops in iteration $t_{\max }$ when all nodes are removed and nodes removed in the iteration step $t_{\max }$ form the Core layer of the network [19].
TABLE 3: Statistical properties of the BTN-CQ and BTN-CD.

\begin{tabular}{lcc}
\hline & BTN-CQ & BTN-CD \\
\hline$\rho$ & $2.49 \%$ & $2.42 \%$ \\
$\langle k\rangle$ & 63.25 & 66.96 \\
$\langle l\rangle$ & 3.10 & 2.76 \\
$C C$ & 0.721 & 0.707 \\
\hline
\end{tabular}

$\rho$ : density; $\langle k\rangle$ : average degree; $\langle l\rangle$ : average shortest path length; $C$ : clustering coefficient.

\section{Results}

3.1. Basic Statistical Properties of BTNs. Table 3 lists the statistical properties of the BTN-CQ and BTN-CD. According to the results, there are no remarkable disparities between the two cities regarding their statistical properties of the BTNs.

\subsection{Types of BTNs}

3.2.1. The Scale-Free Feature. It was found that in the BTNCQ and BTN-CD, there are very few nodes whose degrees are extremely low. Taking BTN-CQ as an example, stations such as Longtousi and Hantu both have a degree of 1 because they are included in only one route which has only two stops. In the real transportation system, Longtousi station plays an important role in distributing passenger flows of the most important railway station in the city of Chongqing, while Hantu station, located in a relatively undeveloped suburban area, is of relevance in the flows of a small number of people along the routes of the shuttle bus. As a result, the two stations are both in extremely low connection conditions and do not conform to the general economic principles. It can be considered that the statistical mechanics for stations like Longtousi and Hantu differ from general stations of BTNs, which are the main part of an urban bus transportation system. Therefore, to reveal the main statistical mechanics of BTNs, the appropriate threshold $\left(k_{\min }\right)$ is valuable to be set up to obtain the objective laws of NDD of the main part of BTNs, in which the degree of nodes exceeds $k_{\min }$. Newman also discussed this situation, "A common situation is that the power-law is obeyed in the tail of the distribution, for large values of $k$, but not in the small- $k$ regime" [39].

In this way, two aspects of the characteristic of BTNs can be found. First, it is impossible to perfectly fit the NDD of BTNs of the two cities with a power-law function or an exponential function in the case of including every node (Figures 9(a1) and 9(a2), Table 4). Second, when the "noise points" (nodes with an extremely low degree) were not considered, the NDD of BTNs could match the power-law distribution well, and the values of correlation coefficient $R^{2}$ of power-law 

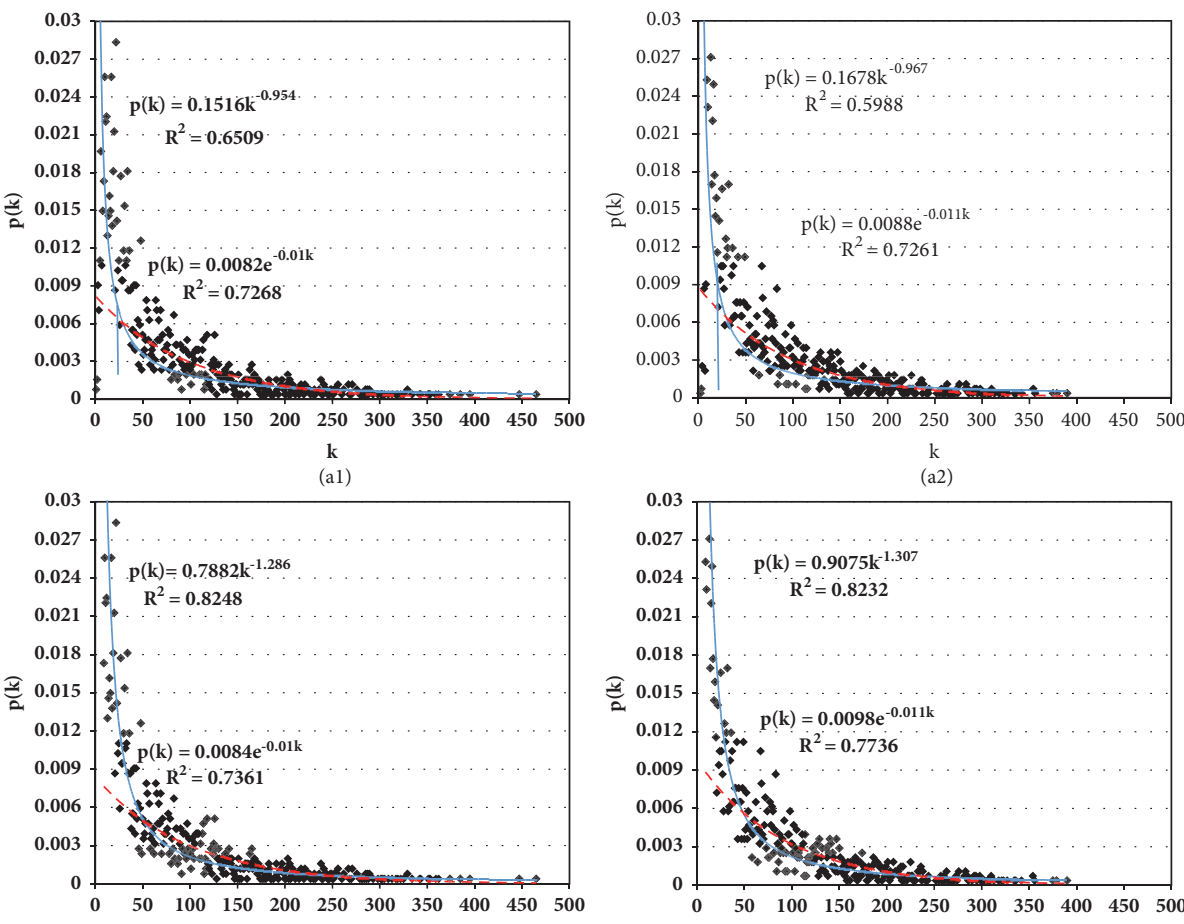

k
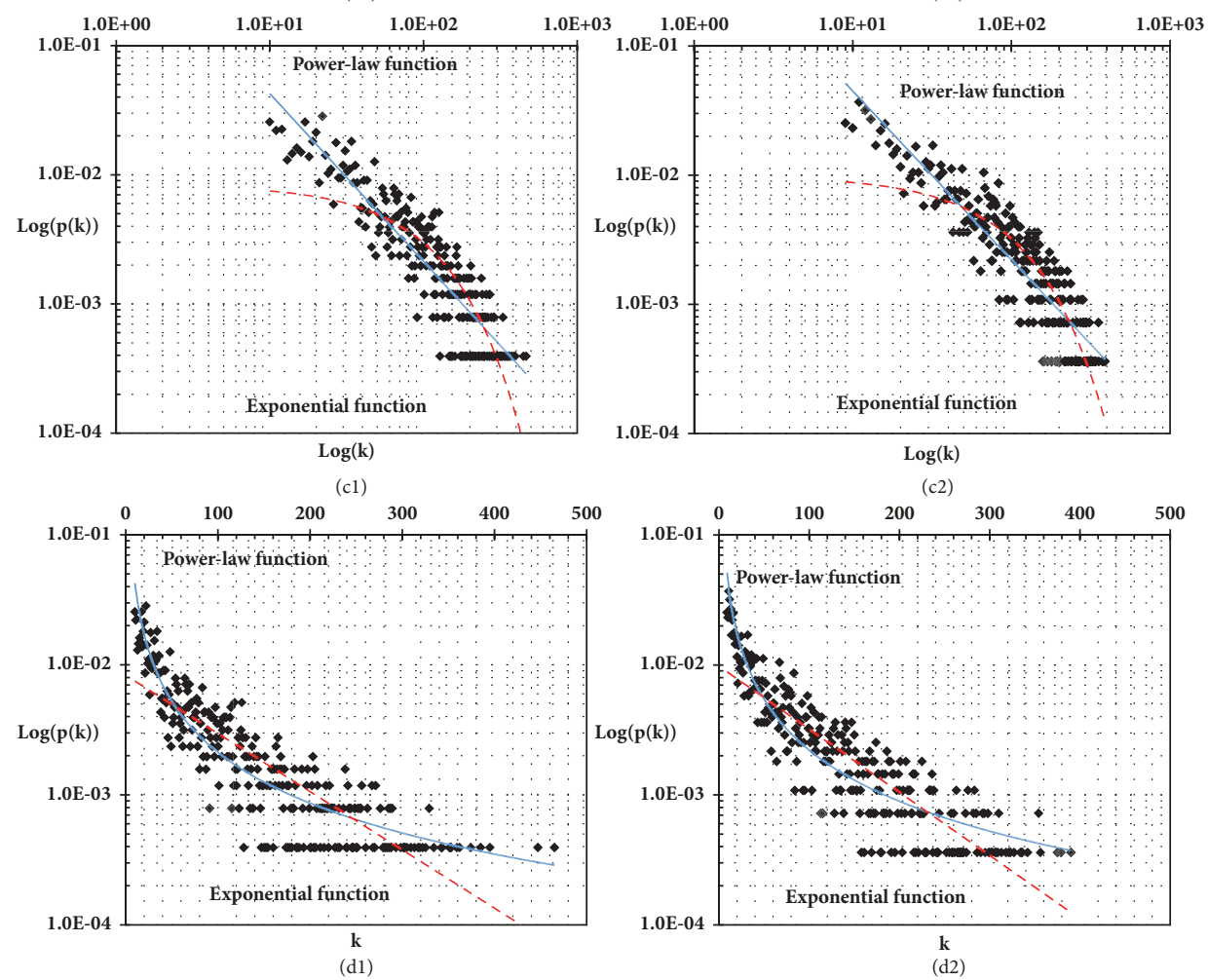

FIGURE 9: Diagrams of curve fitting of BTN-CQ and BTN-CD. (a1) BTN-CQ with "noise points" being included in linear coordinate system; (a2) BTN-CD with "noise points" being included in linear coordinate system; (b1) BTN-CQ with "noise points" being excluded in linear coordinate system; (b2) BTN-CD with "noise points" being excluded in linear coordinate system; (c1) BTN-CQ with "noise points" being excluded in log-log coordinate system; (c2) BTN-CD with "noise points" being excluded in log-log coordinate system; (d1) BTN-CQ with "noise points" being excluded in log-linear coordinate system; (d2) BTN-CD with "noise points" being excluded in log-linear coordinate system. In Figures 9(c1) and 9(c2), "noise points" are shown in the diagrams but not included in the data fitting procedure. In this way, the graphs obviously presented the effect of the "noise points" in the curve fitting process, implying that there might be different statistical mechanics between the "noise points" part and the main part of BTNs. 
TABLE 4: Results of curve fitting of BTN-CQ and BTN-CD.

\begin{tabular}{|c|c|c|c|c|c|c|c|}
\hline BTN & Function & Type & Noise Points & A & $\gamma$ & $\alpha$ & $\mathbf{R}^{2}$ \\
\hline \multirow{2}{*}{ BTN-CQ } & $\mathrm{P}(k)=\mathrm{A} k^{-\gamma}$ & Power & \multirow{4}{*}{ included } & 0.1516 & 0.954 & & 0.6509 \\
\hline & $\mathrm{P}(k)=\mathrm{A} e^{-\alpha k}$ & Exponential & & 0.0082 & & 0.01 & 0.7268 \\
\hline \multirow{2}{*}{ BTN-CD } & $\mathrm{P}(k)=\mathrm{A} k^{-\gamma}$ & Power & & 0.1678 & 0.967 & & 0.5988 \\
\hline & $\mathrm{P}(k)=\mathrm{A} e^{-\alpha k}$ & Exponential & & 0.0088 & & 0.011 & 0.7261 \\
\hline \multirow{2}{*}{ BTN-CQ } & $\mathrm{P}(k)=\mathrm{A} k^{-\gamma}$ & Power & \multirow{4}{*}{ excluded } & 0.7882 & 1.286 & & 0.8248 \\
\hline & $\mathrm{P}(k)=\mathrm{A} e^{-\alpha k}$ & Exponential & & 0.0084 & & 0.01 & 0.7361 \\
\hline \multirow{2}{*}{ BTN-CD } & $\mathrm{P}(k)=\mathrm{A} k^{-\gamma}$ & Power & & 0.9075 & 1.307 & & 0.8232 \\
\hline & $\mathrm{P}(k)=\mathrm{A} e^{-\alpha k}$ & Exponential & & 0.0098 & & 0.011 & 0.7736 \\
\hline
\end{tabular}

TABLE 5: Data on the small-world feature.

\begin{tabular}{lcccc}
\hline BTNs & $\langle\boldsymbol{l}\rangle_{\text {actual }}$ & $<\boldsymbol{l}\rangle_{\text {random }}$ & $\boldsymbol{C C}_{\text {actual }}$ & $\boldsymbol{C C}_{\text {random }}$ \\
\hline BTN-CQ & 3.10 & 2.177 & 0.721 & 0.025 \\
BTN-CD & 2.76 & 2.168 & 0.707 & 0.024 \\
\hline
\end{tabular}

$\mathrm{CC}_{\text {actual }}$ : the clustering coefficient $C C$ of the real bus transportation network; $\langle l\rangle_{\text {actual }}$ : the average shortest path length of the real bus transportation network; $C C_{\text {random }}$ : the clustering coefficient $C C$ of the randomly generated network; $\langle l\rangle_{\text {random }}$ : the average shortest path length of the randomly generated network.

functions were higher than that of exponential functions (Figures 9(b1) and 9(b2), Table 4). Meanwhile, the values of $\mathrm{R}^{2}$ of BTNs were higher than that of the randomly generated BA (Barabási-Albert) model with similar size (Table 2). The results show that the values of $\mathrm{R}^{2}$ for BTNs are 0.8248 (for BTN-CQ) and 0.8232 (for BTN-CD), respectively, which are higher than the value of $\mathrm{R}^{2}$ for BA models (0.8065), indicating that the fitting effect of the power-law function for BTNs is acceptable. The observed relationship in Figure 9 is better fitted by a power-law function rather than an exponential function. The BTN-CQ and BTN-CD are both scale-free networks.

3.2.2. The Small-World Feature. Table 5 shows the comparison of clustering coefficient CC and average shortest path length $\langle l>$ between the real-life BTNs and the network randomly generated, which has the same number of nodes and edges with the real-life BTNs. (Generate a random network 100 times and calculate the arithmetic mean of $C C$ and $<l>$.) Both networks show the small-world phenomenon: $\langle l\rangle_{\text {actual }} \geq\langle l\rangle_{\text {random }}$ but $C C_{\text {actual }} \gg C C_{\text {random }}$ [41].

3.3. Connection Properties of BTNs. Based on the results shown in Figure 10, the transfer convenience of BTN-CD is more efficient than that of BTN-CQ. The probability of linking two stations chosen randomly under the condition of no more than one transfer is $8.06 \%$ lower than that of BTNCD: $18.70 \%$ lower under the condition of no more than two times of transfers, and 5.95\% lower under the condition of no more than three times of transfers, while they share a similar probability of nonstop transportation.

\subsection{Spatial Attributes of BTNs}

3.4.1. Spatial Structure of Degree Distribution. The heat maps, based on degree value and geographical coordinate information of nodes in the BTNs, are drawn, via the online

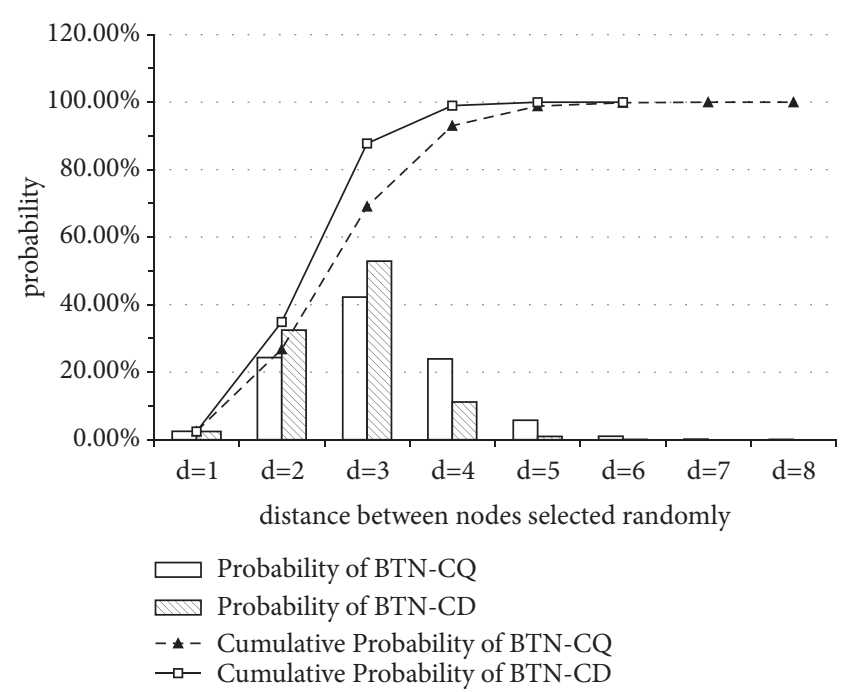

FIgURE 10: Probabilities of distance distribution of BTN-CQ and BTN-CD.

spatial analysis cloud platform GeoHey-https://geohey.com (Figure 11). It shows that, in the city of Chongqing, the relatively high-value region of degree of BTN is mainly concentrated in the center of urban areas, between two large mountains, and notable discontinuous island-like structure can be observed, which corresponds to the clustered multicenter urban groups. Meanwhile, in the city of Chengdu, the areas with relatively high value of degree are evenly scattered in the whole urban areas and continuous planar structures are present, without obvious restricting effects.

For detailed information about spatial properties of BTNs, some quantitative analysis is desirable. The "average degree-distance" distribution function is introduced. The node with the highest degree in the BTN was chosen as the central origin node and all of the other nodes were classified based on their distances from the origin one. The distance 


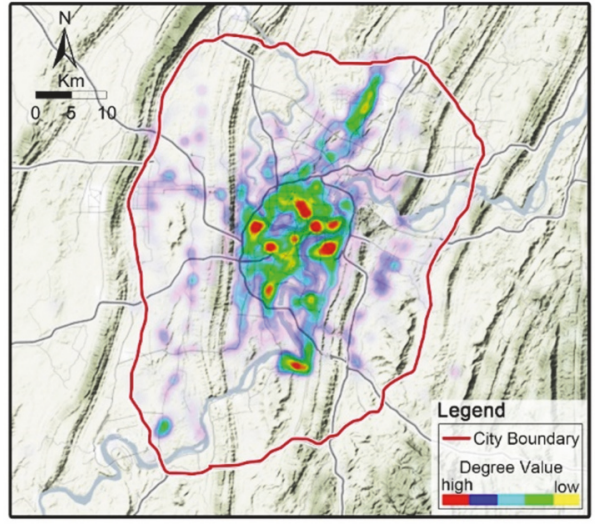

(a)

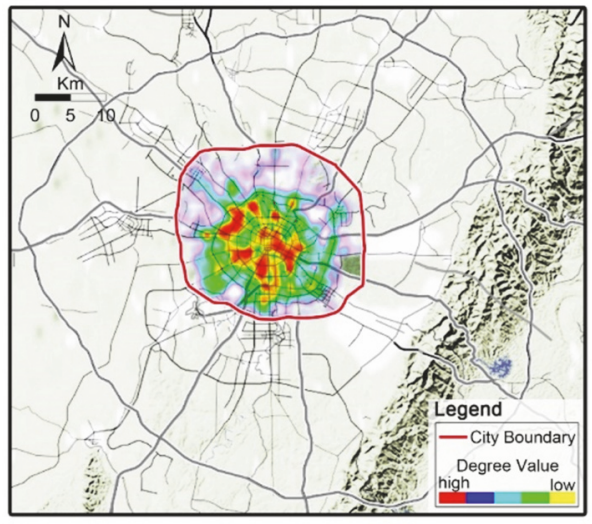

(b)

FIGURE 11: Diagrams of spatial distribution of node degree of (a) BTN-CQ and (b) BTN-CD.

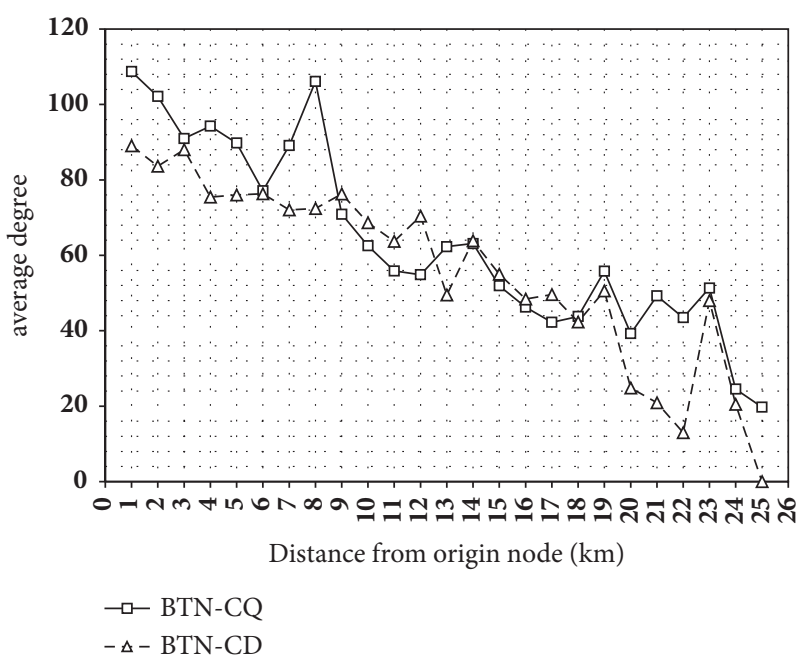

FIGURE 12: Plot of "average degree-distance" distribution function. In both BTNs observed, the nodes within 25 kilometers from the central origin node account for more than $90 \%$, so that the upper limit of the distance observed was set to 25 kilometers. Meanwhile, about half of the sites are distributed within 10 kilometers from the central origin node ( $41.2 \%$ for BTN-CQ, $56.3 \%$ for BTN-CD), so that the area within 10 kilometers from the origin node was mainly analyzed.

interval was set to 1 kilometer. The average degree of nodes in each distance interval was calculated, and the plot of the average degree in each distance interval as a function of distance from the origin node was drawn (Figure 12).

It can be seen from Figure 12, both of the BTNs show a general downward trend, which means that as the distance from the central node increases, the average value of the nodes in each distance interval gradually decreases. However, the two BTNs also show significant differences:

(a) Although the average degree of BTN-CQ is lower than that of BTN-CD (63.25 and 66.96, respectively), in the within-10km areas of the origin node, the average degrees of BTN-CQ in almost every distance interval are significantly higher than that of BTN-CD. (b) In the within-10km area of BTN-CD, as the distance from the central origin node increases, the average degrees show, roughly speaking, a downward trend. As for BTN$\mathrm{CQ}$, in the range of $6-\mathrm{km}$ to $8-\mathrm{km}$, there are two relatively high values of average degree emerging. In particular, in the distance interval of $7-\mathrm{km}$ to $8-\mathrm{km}$, the average value reaches 106.13, which can be regarded as being equivalent to the highest value of 108.72 in the most central area, located within $1 \mathrm{~km}$ from the origin node in BTN-CQ.

Those different spatial properties indicate that in the BTN-CQ, there are more than one hub centers in the spatial structure of network, implying that more than one spatial hub area exist, even relatively far away from the central hubs. Relatively, the single-center feature of the spatial structure of BTN-CD is more significant.

As a result, the relationships between the two BTNs' spatial structures and urban geographical environment demonstrate that the development and evolution of urban BTN topology are strongly affected by the urban spatial geographic conditions and spatial morphology.

3.4.2. Spatial Structure of Core Layer Distribution. In the BTN-CQ, there are 205 bus stations in the Core layer, 8.1\% of the total. As for BTN-CD, the number of stations and percentage in the Core layer are 557 and 20.1\%, respectively. There are fewer stations in the Core layer in Chongqing than in Chengdu. Figure 13 shows, in the BTN-CQ, stations in the Core layer are clustered in the center of the city, between two large mountains. In Chengdu, nodes in the Core layer are sparsely distributed in the whole urban area. The difference in node distribution between Core layers of two BTNs also indicates that urban geographic conditions and spatial morphology affect the topology structure of BTNs.

In order to know how the BTNs are organized in the process of development, the assortativity analyses of $\mathrm{k}$ core were conducted (Table 6). The concept of assortativity was introduced by Newman in 2002 [44] and has been extensively studied since then. It represents to what extent nodes in a network associate with other nodes in the network, being of similar sort or being opposing sort. Generally, the 
TABLE 6: Assortativity $\rho_{D}$ of nodes observed based on k-core layers.

\begin{tabular}{|c|c|c|c|c|}
\hline \multirow{2}{*}{$\begin{array}{l}-- \\
\text { layers }\end{array}$} & \multicolumn{2}{|r|}{ BTN-CQ } & \multicolumn{2}{|r|}{ BTN-CD } \\
\hline & $\rho_{D}$ & number of nodes observed & $\rho_{D}$ & number of nodes observed \\
\hline Core layer & -0.011 & 205 & -0.007 & 557 \\
\hline$k$-core $=65$ & -0.004 & 403 & -0.010 & 624 \\
\hline$k$-core $=60$ & -0.011 & 548 & -0.018 & 720 \\
\hline $\mathrm{k}$-core $=55$ & 0.000 & 620 & 0.027 & 917 \\
\hline $\mathrm{k}$-core $=\mathbf{5 0}$ & 0.005 & 758 & 0.034 & 1003 \\
\hline$k$-core $=45$ & 0.022 & 911 & 0.035 & 1092 \\
\hline $\mathrm{k}$-core $=\mathbf{4 0}$ & 0.030 & 1043 & 0.039 & 1207 \\
\hline $\mathrm{k}$-core $=35$ & 0.040 & 1130 & 0.043 & 1359 \\
\hline $\mathbf{k}$-core $=\mathbf{3 0}$ & 0.047 & 1289 & 0.056 & 1540 \\
\hline k-core $=25$ & 0.072 & 1510 & 0.079 & 1760 \\
\hline $\mathrm{k}$-core $=\mathbf{2 0}$ & 0.107 & 1767 & 0.095 & 1919 \\
\hline $\mathrm{k}$-core $=15$ & 0.133 & 2026 & 0.129 & 2232 \\
\hline $\mathrm{k}$-core $=10$ & 0.154 & 2297 & 0.155 & 2621 \\
\hline$k$-core $=5$ & 0.167 & 2492 & 0.159 & 2756 \\
\hline$k$-core $=1$ & 0.167 & 2539 & 0.159 & 2766 \\
\hline
\end{tabular}

$\mathrm{k}$-core $=\mathrm{n}$ means nodes observed are in the levels of which $\mathrm{k}$-shell is greater than or equal to $\mathrm{n}$; in particular, $\mathrm{k}$-core $=1$ means nodes observed are all of the nodes in BTNs [30, 31].

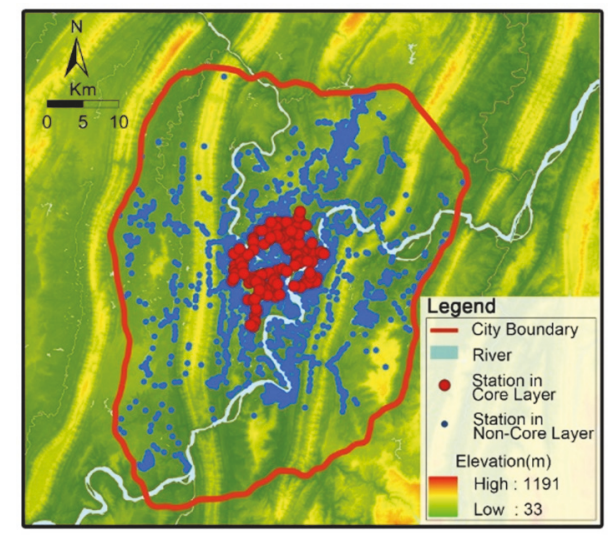

(a)

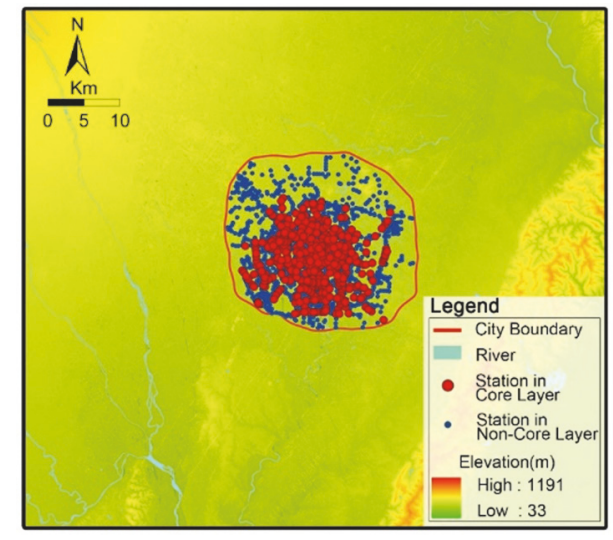

(b)

FIGURE 13: Diagrams of spatial distribution of Core layer of (a) BTN-CQ and (b) BTN-CD.

assortativity of a network is determined for the degree of the nodes in the network. The degree assortativity $\rho_{\mathrm{D}}$ is determined as follows $[44,45]$ :

$$
\rho_{\mathrm{D}}=\frac{\sum_{\mathrm{jk}} \mathrm{jk}\left(\mathrm{e}_{\mathrm{jk}}-\mathrm{q}_{\mathrm{j}} \mathrm{q}_{\mathrm{k}}\right)}{\sigma_{\mathrm{q}}^{2}}
$$

where $e_{j k}$ is the joint-remaining degree probability for remaining degree $j$ and remaining degree $k$ (the remaining degree of a node is equal to the degree of that node minus one); $\mathrm{q}_{\mathrm{k}}=(\mathrm{k}+1) \mathrm{p}_{\mathrm{k}+1} / \sum_{\mathrm{j}} \mathrm{j}_{\mathrm{j}}$ is the normalized distribution of the remaining degree $\mathrm{D}_{\mathrm{r}}$ of a randomly selected node; $\sigma_{\mathrm{q}}$ is the standard deviation of $q_{k}$.

Observing the assortativity indicators of different k-core levels, we found that the two BTNs showed some common characteristics: when the stations observed are in the relatively higher k-core level, the BTNs show disassortative characteristics, implying that, in those layers, bus stations with higher degree tend to connect to the stations with relatively lower degrees. As for the nodes in the relatively lower k-core layers, the assortative features appear in the BTNs, which means that the bus stations in those layers turn to connect to stations with similar degrees to themselves. The mechanism may be that the reasonable passenger-load of bus routes is significant for the transportation systems.

When the stations observed are in the relatively higher layer, the ability of a single station to attract passengers is relatively stronger. In order to avoid bus routes overload, which will cause adverse effects on the comfort and safety of the transportation system. In contrast, when the k-core level reduced, roughly speaking, the ability of a single station to attract passengers is reduced, too. In order to enhance the attraction of bus routes to passengers, relatively highlevel stations tend to connect with other high-level stations to 
TABLE 7: Scale-free networks with $\gamma \leq 2$.

\begin{tabular}{lccc}
\hline Network of & $\mathbf{n}$ & $\mathbf{m}$ & $\gamma$ \\
\hline Software packages [32] & 1439 & 1723 & \\
\hline Email messages [32] & 59912 & 86300 & \\
\hline Ythan [33] & 135 & 596 & $1.6 / 1.4$ \\
\hline Silwood Park [33] & 153 & 365 & $1.5 / 2.0$ \\
\hline E-mail [34] & 56969 & 84314 & 1.13 \\
\hline Co-authorship in HEP [35] & 56627 & 9796471 & 1.81 \\
\hline Restricted Word Web [36] & 478773 & $1.77 * 10^{7}$ & 1.2 \\
\hline
\end{tabular}

$\mathrm{n}$ : the number of nodes; m: the number of edges; $\gamma$ : the power-law exponent.

improve the overall attractiveness of bus routes, which cause the whole networks assortative.

3.5. Summary of Results. There are apparent differences regarding areas, geographic conditions, and spatial morphology between Chongqing and Chengdu (Section 2.2). Despite these differences, there are also many similarities between the BTNs of the two cities in terms of basic statistical properties and types of networks. These findings, such as statistical indicators, scale-free features, and smallworld features, are consistent with previous studies [14, 23]. These results indicate that there can be a general law in the statistical mechanics of BTNs. In-depth observations of connection properties and spatial attributes of the BTNs reveal enormous disparities between the two cities. For BTN$\mathrm{CD}$, the transfer convenience is more efficient than that of BTN-CQ (Section 3.3). Regarding the spatial structures of networks, BTN-CQ presents discontinuity with several local centers, while BTN-CD shows continuously relatively even distribution. This difference indicates that there are mechanisms of dynamic development regarding urban spatial factors.

\section{Discussion}

Based on the analysis results in Section 3.2, the exponents $\gamma$ of both BTNs are less than 2, which is rather small (1.286 for BTN-CQ and 1.307 for BTN-CD, shown in Table 4) and thus smaller than the value of the BA framework with the number of edges and nodes corresponding to the real networks (1.922 for BA framework, shown in Table 2). For the public transportation networks in the real environment, BTN-CQ and BTN-CD include two additional features: (a) economical reasonableness: the investment of building a bus station is relatively high, and many bus routes through it are needed to ensure the economic rationality of the transportation system; (b) accessibility to new routes: it is costly to establish a bus station, but it is costless to link an established one to improve the system. The above facts lead to the following two aspects about BTNs, which constitute the reason why the exponents of BTNs are different from the BA framework: (a) the number of bus stations with really low connections is relatively small; (b) the number of hubs in high-connection conditions is relatively high.
Literature review shows that several real-world networks with power-law degree distributions have exponents $\gamma$ in the range $2 \leq \gamma \leq 3[32,39,46-48]$, while there are also quite a few scale-free real-world networks with exponents $\gamma$ in the range $1 \leq \gamma \leq 2$, which have received less attention, despite their widespread appearance (Table 7).

Researchers believed that such kinds of networks are relatively more dense, which means there are a larger number of edges, a larger average degree, and more hubs in the structure of networks.

A key characteristic of this type of networks is that their average degree grows with the system size, which suggests that making a link is inexpensive [49]. As a software package, it is costly to make a package, but it is costless to use an existing one [49]. As for BTNs, the development mechanism of the bus transportation system is similar to the software case. In summary, the similar dynamic mechanism results in a relatively larger number of hub nodes and a relatively small power exponent $\gamma$ for BTNs.

Based on the analysis in Sections 3.3 and 3.4, this study concludes that the statistical mechanic "spatial matching" leads to the considerable differences in the connection properties and spatial attributes of the BTNs. With regard to connection properties, Chongqing has a less efficient BTNs for transfers, which is attributed to the large coverage of the area and relatively sparser bus stations, as well as the geographical barriers, including mountains and rivers. Furthermore, the topological structure of BTN-CQ has discontinuous multiple centers, which matches the characteristics that Chongqing has a clustered multicenter urban spatial structure. At the same time, the topological structure of BTN-CD has a continuous distributed spatial structure, which matches the characteristics that Chengdu has a single-center and ringlayer urban spatial structure, with relatively few geographical barriers.

The power-law distribution is well followed for the main part of BTN-CQ and BTN-CD (Section 3.2.1), which indicates that there are two rules in the statistical mechanics of BTNs, "growth" and "preferential connection", proposed by Barabási et al. [26, 28]. The processes of generation and development of an urban transportation system always start from scratch, and stations and routes increase step by step, demonstrating the rule of "growth". At the same time, it is often the case that when a new node links into the network, there is a tendency to preferentially target an 
existing stop in better connection condition, following the rule of "preferential connection", which conforms to the principle of economic efficiency in the development process of BTNs.

Based on the analysis in Section 3.2.1. (Figure 9), there is also a rule in the statistical mechanics "practical adaptability", revealing that not all the stations jointed into the bus transportation systems were linked to stations which are well connected in the BTNs. The principle of economic effectiveness will be followed in most cases. However, in a real BTN, certain special stops are needed to meet the practical demand in certain areas or particular circumstances, such as Longtousi station and Hantu station in BTN-CQ.

Meanwhile, the proportion of such stations will be limited by the principle of economic effectiveness. Therefore, there are only a few stations that fail to match the mechanic "preferential connection" but follow the rule "practical adaptability". The mechanic "practical adaptability" interprets the appearance of "noise points" in the fitting process for powerlaw function (Figures 9(c1) and 9(c2)). These stations, less in quantity and characterized by extremely low degree, present the mechanic "practical adaptability" rather than "preferential connection" in the process of BTN development.

In summary, the statistical mechanics of "spatial matching", "growth", "preferential connection", and "practical adaptability" lead to the similarities and differences of the spatial and topological properties of BTNs in Chongqing and Chengdu.

\section{Conclusions}

BTNs of Chongqing and Chengdu, which are different in terms of geographic conditions and spatial morphology, are considered as the research objects in this study. Their basic statistical properties, types, connection properties, and spatial attributes are compared through the use of complex network theory and spatial analysis method. The major findings can be summarized according to the following three aspects:

(a) For the topology structures of BTNs in Chongqing and Chengdu, there is a notable disparity in spatial structures that matches well with the urban geographic conditions and spatial morphology. The case study shows that BTN of the mountainous city, Chongqing, which has clustered multiple centers, presents a discontinuous spatial islandshape characteristic, while BTN of plains city, Chengdu, which has just one single-center and ring-layer structure, shows continuous spatial distribution characteristic.

(b) BTNs of Chongqing and Chengdu are found to comply with the power-law distribution upon the removal of small amounts of special stops that have extremely low degrees. This kind of stops, needed in special urban areas, do not comply with the principle of economic efficiency. It is necessary to distinguish the roles and mechanisms of these special stops in the analysis of networks because the roles and mechanisms of them are different from the major part of BTNs.

(c) The statistical mechanics of urban bus system of Chongqing and Chengdu are found in four aspects, namely, "spatial matching", "growth", "preferential connection", and "practical adaptability".

Further research to understand the internal rules and formation mechanisms of real PTNs is suggested. It could enhance the understanding of PTNs by properly analyzing both topological and spatial attributes of networks. Practically, this paper reveals the rules of variation and development of urban BTNs in the spatial context, providing theoretical strategies for the optimization and planning of infrastructure and land utilization. This work also has limitations, particularly regarding the small sample size. In further studies, priority can be placed on hybrid transportation networks including bus and metro to develop strategies for hybrid networks engaged in spatial structures.

\section{Data Availability}

The datasets generated and analyzed during the current study are available from the corresponding author on reasonable request.

\section{Conflicts of Interest}

The authors declare no conflicts of interest.

\section{Acknowledgments}

This work was supported by the National Science \& Technology Pillar Program during the 12th Five-Year Plan Period [grant number: 2013BAJ13B07]; Chongqing Municipal Special Project on Scientific \& Technological Innovation of Social Undertakings \& People's Livelihood Security [grant numbers: cstc2017shms-zdyfX0026 and cstc2016shmszx30001]; and Chongqing Municipal Graduate Research \& Innovation Project [grant number: CYB17028]. We also wish to thank Papergoing and AJE for their linguistic assistance during the preparation of this manuscript.

\section{References}

[1] A. A. De Bona, K. V. O. Fonseca, M. O. Rosa, R. Lüders, and M. R. B. S. Delgado, "Analysis of Public Bus Transportation of a Brazilian City Based on the Theory of Complex Networks Using the P-Space," Mathematical Problems in Engineering, vol. 2016, Article ID 3898762, 12 pages, 2016.

[2] S. Wandelt and X. Sun, "Evolution of the international air transportation country network from 2002 to 2013," Transportation Research Part E: Logistics and Transportation Review, vol. 82, pp. 55-78, 2015.

[3] A. Huang, H. M. Zhang, W. Guan, Y. Yang, and G. Zong, "Cascading failures in weighted complex networks of transit systems based on coupled map lattices," Mathematical Problems in Engineering, vol. 2015, Article ID 940795, 2015.

[4] A. Chatterjee, G. Ramadurai, and K. Jagannathan, "Contagion processes on urban bus networks in Indian cities," Complexity, vol. 21, no. S2, pp. 451-458, 2016.

[5] J. Lin and Y. Ban, "Comparative analysis on topological structures of urban street networks," ISPRS International Journal of Geo-Information, vol. 6, no. 10, 2017. 
[6] O. Cats, G.-J. Koppenol, and M. Warnier, "Robustness assessment of link capacity reduction for complex networks: Application for public transport systems," Reliability Engineering \& System Safety, vol. 167, pp. 544-553, 2017.

[7] J. Zhu, Y. Nie, H. Gao, F. Liu, and L. Yu, "GIS-based visibility network and defensibility model to reconstruct defensive system of the Han Dynasty in Central Xinjiang, China," ISPRS International Journal of Geo-Information, vol. 6, no. 8, 2017.

[8] F. Morone and H. A. Makse, "Influence maximization in complex networks through optimal percolation," Nature, vol. 524, no. 7563, pp. 65-68, 2015.

[9] J. Ruths and D. Ruths, "Control profiles of complex networks," American Association for the Advancement of Science: Science, vol. 343, no. 6177, pp. 1373-1376, 2014.

[10] C. Zhou, L. Ding, M. J. Skibniewski, H. Luo, and S. Jiang, "Characterizing time series of near-miss accidents in metro construction via complex network theory," Safety Science, vol. 98, pp. 145-158, 2017.

[11] A. R. Benson, D. F. Gleich, and J. Leskovec, "Higher-order organization of complex networks," Science, vol. 353, no. 6295, pp. 163-166, 2016.

[12] J. Ruths and D. Ruths, "Response to comment on "Control profiles of complex networks", Science, vol. 346, no. 6209, pp. 561-d, 2014.

[13] C. Campbell, K. Shea, and R. Albert, "Comment on "control profiles of complex networks"," Science, vol. 346, no. 6209, p. 561, 2014.

[14] X. Zhang, G. Chen, Y. Han, and M. Gao, "Modeling and analysis of bus weighted complex network in Qingdao city based on dynamic travel time," Multimedia Tools and Applications, vol. 75, no. 24, pp. 17553-17572, 2016.

[15] S. Feng, B. Hu, C. Nie, X. Shen, and Y. Ci, "Empirical investigation of topological and weighted properties of a bus transport network from China," Chinese Physics B, vol. 25, no. 3, p. 030504 , 2016.

[16] X.-H. Yang, G. Chen, S.-Y. Chen, W.-L. Wang, and L. Wang, "Study on some bus transport networks in China with considering spatial characteristics," Transportation Research Part A: Policy and Practice, vol. 69, pp. 1-10, 2014.

[17] J. Sienkiewicz and J. A. Hołyst, "Statistical analysis of 22 public transport networks in Poland," Physical Review E: Statistical, Nonlinear, and Soft Matter Physics, vol. 72, no. 4, Article ID 046127, 2005.

[18] S. Dunn and S. M. Wilkinson, "Increasing the resilience of air traffic networks using a network graph theory approach," Transportation Research Part E: Logistics and Transportation Review, vol. 90, pp. 39-50, 2016.

[19] W.-B. Du, X.-L. Zhou, O. Lordan, Z. Wang, C. Zhao, and Y.B. Zhu, "Analysis of the Chinese Airline Network as multilayer networks," Transportation Research Part E: Logistics and Transportation Review, vol. 89, pp. 108-116, 2016.

[20] O. Lordan, J. M. Sallan, P. Simo, and D. Gonzalez-Prieto, "Robustness of the air transport network," Transportation Research Part E: Logistics and Transportation Review, vol. 68, pp. 155-163, 2014.

[21] J. Feng, X. Li, B. Mao, Q. Xu, and Y. Bai, "Weighted Complex Network Analysis of the Different Patterns of Metro Traffic Flows on Weekday and Weekend," Discrete Dynamics in Nature and Society, vol. 2016, 2016.

[22] P. Sen, S. Dasgupta, A. Chatterjee, P. A. Sreeram, G. Mukherjee, and S. S. Manna, "Small-world properties of the Indian railway network," Physical Review E: Statistical, Nonlinear, and Soft Matter Physics, vol. 67, no. 3, Article ID 036106, 5 pages, 2003.

[23] D. Tsiotas and S. Polyzos, "Analyzing the Maritime Transportation System in Greece: a Complex Network Approach," Networks and Spatial Economics, vol. 15, no. 4, pp. 981-1010, 2015.

[24] Y. Sun, L. Mburu, and S. Wang, "Analysis of community properties and node properties to understand the structure of the bus transport network," Physica A: Statistical Mechanics and its Applications, vol. 450, pp. 523-530, 2016.

[25] B. Hu, S. Feng, and C. Nie, "Bus transport network of Shenyang considering competitive and cooperative relationship," Physica A: Statistical Mechanics and its Applications, vol. 466, pp. 259268, 2017.

[26] R. Albert, H. Jeong, and A.-L. Barabási, "Error and attack tolerance of complex networks," Nature, vol. 406, no. 6794, pp. 378-382, 2000

[27] M. Barthélemy, "Spatial networks," Physics Reports, vol. 499, no. 1-3, pp. 1-101, 2011.

[28] A. Barabasi and R. Albert, "Emergence of scaling in random networks," Science, vol. 286, no. 5439, pp. 509-512, 1999.

[29] A. L. Barabási, R. Albert, and H. Jeong, "Mean-field theory for scale-free random networks," Physica A: Statistical Mechanics and its Applications, vol. 272, no. 1, pp. 173-187, 1999.

[30] S. Carmi, S. Havlin, S. Kirkpatrick, Y. Shavitt, and E. Shir, "A model of Internet topology using k-shell decomposition," Proceedings of the National Acadamy of Sciences of the United States of America, vol. 104, no. 27, pp. 11150-11154, 2007.

[31] V. Batagelj and M. Zaversnik, "An O(m) Algorithm for Cores Decomposition of Networks," Computer Science, vol. 1, no. 6, pp. 34-37, 2003.

[32] M. E. J. Newman, "The structure and function of complex networks," SIAM Review, vol. 45, no. 2, pp. 167-256, 2003.

[33] W. J. Xiao, W. D. Chen, and B. Parhami, "On necessary conditions for scale-freedom in complex networks, with applications to computer communication systems," International Journal of Systems Science, vol. 42, no. 6, pp. 951-958, 2011.

[34] H. Ebel, L.-I. Mielsch, and S. Bornholdt, "Scale-free topology of e-mail networks," Physical Review E: Statistical, Nonlinear, and Soft Matter Physics, vol. 66, no. 3, Article ID 035103, 2002.

[35] M. E. J. Newman, "Scientific collaboration networks. II. Shortest paths, weighted networks, and centrality," Physical Review E: Statistical Physics, Plasmas, Fluids, and Related Interdisciplinary Topics, vol. 64, no. 1, p. 7, 2001.

[36] R. F. I. Cancho and R. V. Solé, "The small world of human language," Proceedings of the Royal Society B Biological Science, vol. 268, no. 1482, pp. 2261-2265, 2001.

[37] S. Derrible and C. Kennedy, "Applications of graph theory and network science to transit network design," Transport Reviews, vol. 31, no. 4, pp. 495-519, 2011.

[38] A. Hagberg, P. Swart, and D. S. Chult, Exploring network structure, dynamics, and function using networkx, Los Alamos National Lab, 2008.

[39] M. E. J. Newman, Networks: An Introduction, Oxford University Press, Oxford, UK, 2010.

[40] J. Feng, X. Li, B. Mao, Q. Xu, and Y. Bai, "Weighted complex network analysis of the Beijing subway system: Train and passenger flows," Physica A: Statistical Mechanics and its Applications, vol. 474, pp. 213-223, 2017.

[41] D. J. Watts, "Collective dynamics of 'small-world' networks," Nature, vol. 393, no. 6684, pp. 440-442, 1998. 
[42] T. Verma, N. A. Araújo, and H. J. Herrmann, "Revealing the structure of the world airline network," Scientific Reports, vol. 4, no. 1, 2015.

[43] S. N. Dorogovtsev, A. V. Goltsev, and J. F. Mendes, "k-core organization of complex networks," Physica D: Nonlinear Phenomena, vol. 224, no. 1-2, pp. 7-19, 2006.

[44] M. E. Newman, "Mixing patterns in networks," Physical Review Letters, vol. 89, no. 20, 2002.

[45] R. Noldus and P. Van Mieghem, "Assortativity in complex networks," Journal of Complex Networks, vol. 3, no. 4, pp. 507542, 2015.

[46] R. Albert and A. Barabási, "Statistical mechanics of complex networks," Reviews of Modern Physics, vol. 74, no. 1, pp. 47-97, 2002.

[47] S. H. Strogatz, "Exploring complex networks," Nature, vol. 410, no. 6825 , pp. 268-276, 2001.

[48] M. E. J. Newman, "Power laws, Pareto distributions and Zipf's law," Contemporary Physiscs, vol. 46, no. 5, pp. 323-351, 2005.

[49] H. Seyed-Allaei, G. Bianconi, and M. Marsili, "Scale-free networks with an exponent less than two," Physical Review E: Statistical, Nonlinear, and Soft Matter Physics, vol. 73, no. 4, 2006. 


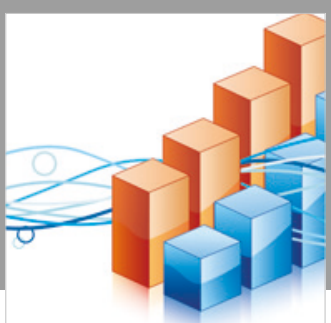

Advances in

Operations Research

\section{-n-m}
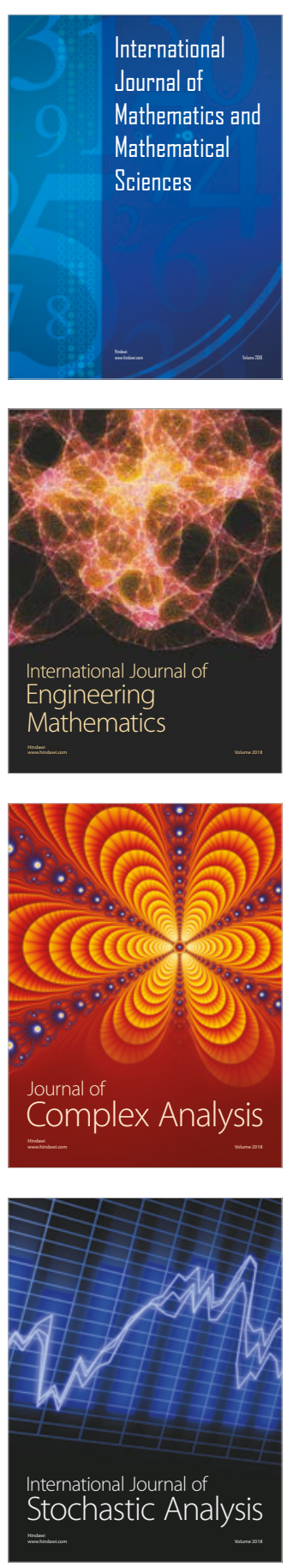
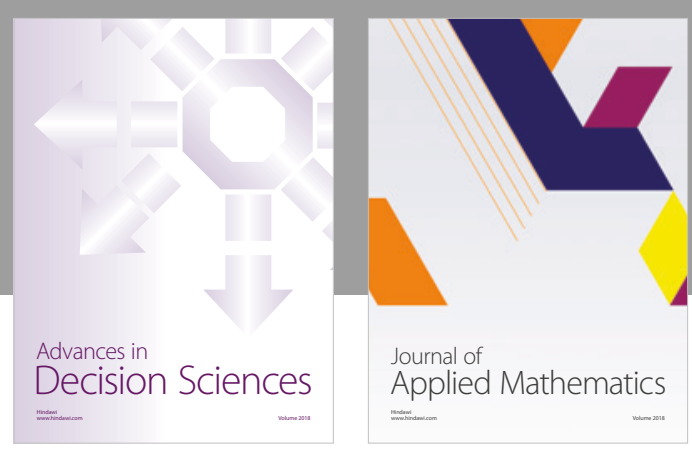

Journal of

Applied Mathematics
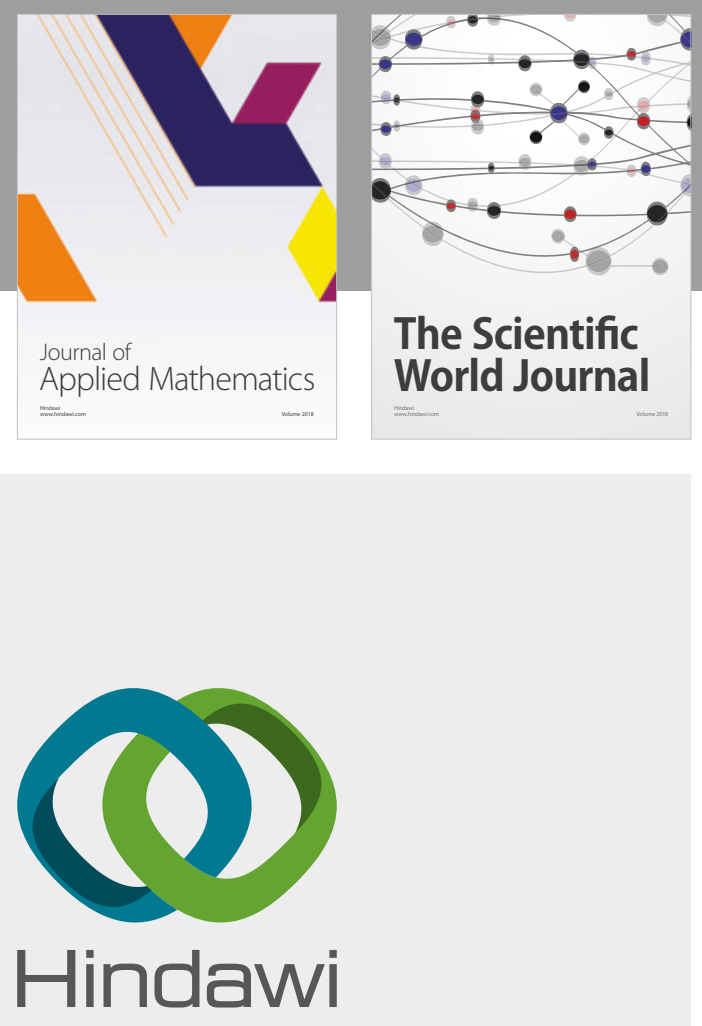

Submit your manuscripts at

www.hindawi.com

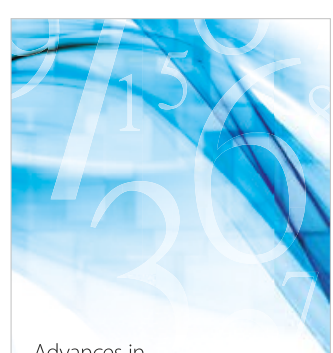

Advances in
Numerical Analysis
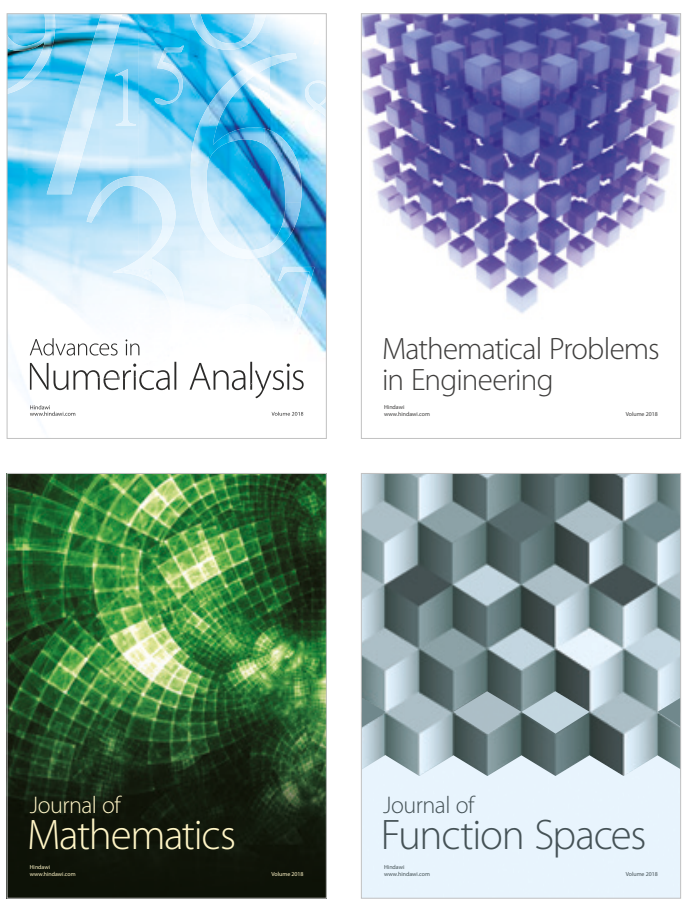

Mathematical Problems in Engineering

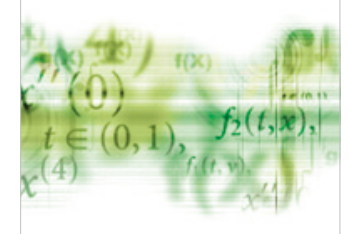

International Journal of

Differential Equations

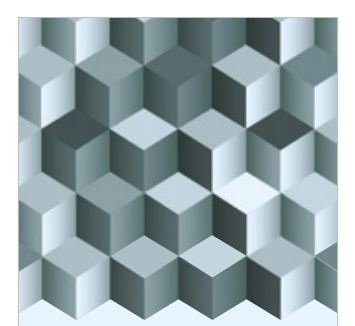

Journal of

Function Spaces
The Scientific

World Journal

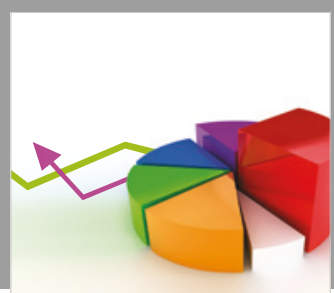

Journal of

Probability and Statistics
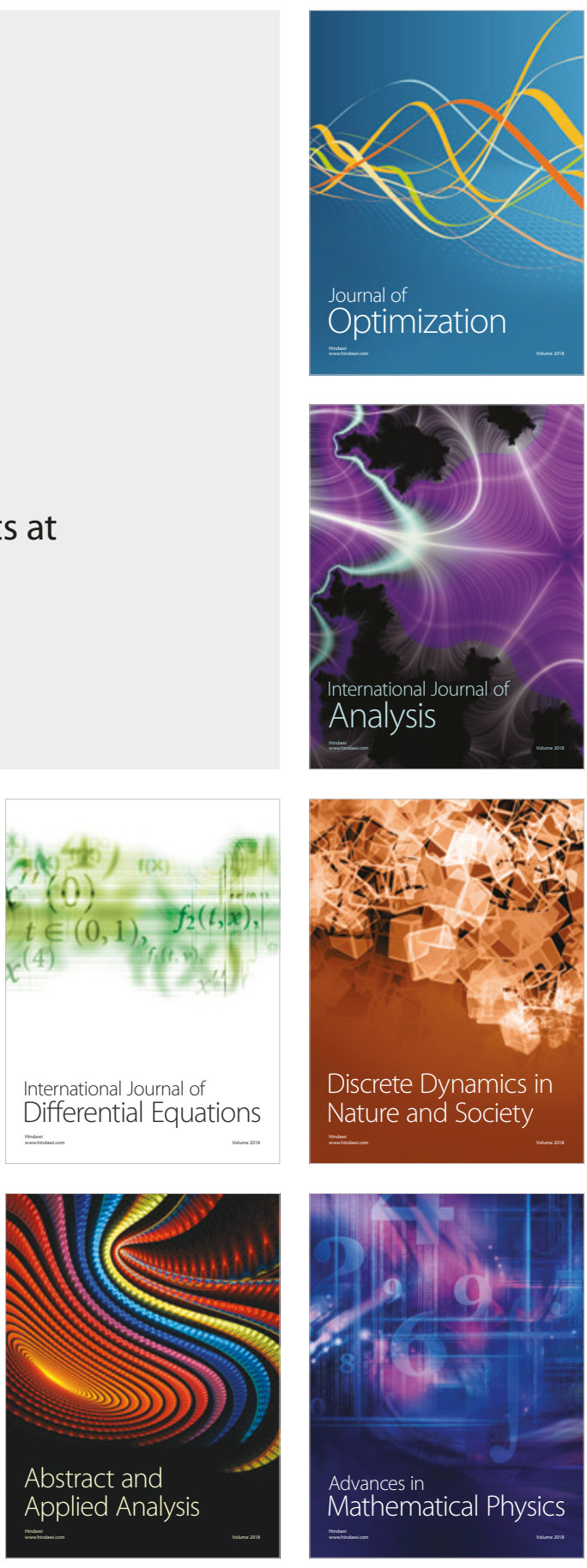